\title{
No evidence that magnification devices improve the success of endodontic therapy
}

\author{
Abstracted from \\ Del Fabbro M, Taschieri S, Lodi G, Banfi G, Weinstein RL. \\ Magnification devices for endodontic therapy. Cochrane Database Syst Rev 2015; 12: CD005969. \\ doi: 10.1002/14651858.CD005969.pub3. Review. PMID:26650099 \\ Address for correspondence: Massimo Del Fabbro, Department of Biomedical, Surgical and Dental Sciences, \\ Universita degli Studi di Milano IRCCS Galeaazzi 4, Orthopaedic Institute Via Riccardo Galeazzzi 4, Milan, 20161 Italy. \\ E-mail: Massimo.delfabbro@unimi.it
}

\section{Question: Does the use of magnification devices in endodontic treatment increase outcome success?}

Data sources Cochrane Oral Health group Trials Register, the Cochrane Central Register of Controlled Trials, Medline, US National Institutes of Health Trials Register, WHO-Clinical Trials Registry Platform for ongoing trials.

Study selection Only randomised and quasi-randomised studies were pursued. No restrictions were placed on language or date of publication. The primary outcome sought was treatment success (complete healing or incomplete healing), uncertain healing and failure after one year of treatment, between one and four years and more than four years after treatment. Secondary outcomes considered for the inclusion criteria included outcomes related to the advantage of using a given magnification device in the clinical procedure such as; greater accuracy, the ease of removing broken instruments from the canal, quality of visualisation, quality of root end filling for the retrograde treatment, quality of perforation repair and the total time required for completing the clinical procedure..

Data extraction and synthesis Data would have been extracted by two review authors independently using a standardised data extraction form, and any disagreement would have been resolved by discussion and a third reviewer would have been consulted. Two review authors would have independently undertaken an assessment of the risk of bias.

Results The searches retrieved 1,234 studies. None of these satisfied the selection criteria, therefore no analysis was completed.

Conclusions No article was identified in the current literature for the review that satisfied the inclusion criteria. It is unknown if and how the type of magnification device affects the treatment outcome considering the high number of factors that may have a significant impact on the success of endodontic therapy.

This paper is based on a Cochrane Review published in the Cochrane Library 2015, issue 10 (see www.thecochranelibrary.com for information). Cochrane Reviews are regularly updated as new evidence emerges and in response to feedback, and the Cochrane Library should be consulted for the most recent version of the review.

\section{Commentary}

The technological progress and advances available today have transformed dental practice. Magnification devices have become widely used and are especially useful when providing endodontic treatment. Magnification loupes and microscopes are commonly and routinely used nowadays providing dentists ease of care with increased visibility and illumination. In the mid-1990s the microscope was first introduced in dentistry and was only infrequently used. Today almost all endodontists are using microscopes in nonsurgical and surgical endodontic treatment. A questionnaire showed that the use of the operating microscope by endodontists increased from 52\% in 1999 to $90 \%$ in $2007 .{ }^{1}$

Many dentists believe that magnification is the single most effective tool they use and that the higher the magnification the better treatment they can provide.

In this Cochrane review (an update to the 2009 review) the authors seek to evaluate studies that can provide evidence to support these claims. The question in particular addresses endodontic care and asks; 'do we really need magnification devices to improve the success of root canal treatments (endodontic therapy)'? The objective was to evaluate studies to assess the primary outcome of endodontic success with magnification use $v s$. without magnification use. Equipment preferences on particular clinical situations and the total time required were considered as secondary outcomes.

Although studies were identified, the authors could not include any. Of the 1,234 , three were identified as possible inclusions only to be excluded after further evaluation was done by all of the review authors. The three were excluded based on weaknesses discovered in methodologies and participant selection. Two were not randomised and treatment used different techniques and equipment.

The authors concluded that well designed research is still needed to evaluate that the use of magnification is a significant advantage for the success of endodontic therapy.

The secondary outcomes mentioned may also have significant relevance and be worthy of further review. For example, in a RCT on treatment time, published in 2015, the authors were able to conclude that the use of a magnification loupe can significantly reduce chair-time in endodontic therapy. The study reports the result that magnification reduces treatment time by $20-24 \%(95 \%$ CI, P <0.001). ${ }^{2}$

The same author (Fabbro) published a systematic review in 2010 in the Journal of Dentistry. The topic was to investigate the use of 
magnification devices and their impact in clinical and radiographic outcomes, and included three prospective studies dealing with endodontic surgery and concluded that the type of magnification device per se can only minimally affect the treatment outcome. ${ }^{3}$

It may seem obvious that the use of magnification could only be advantageous, thus increasing outcome success in dental practice, however, finding relevant studies that can effectively show this are still difficult to reach
Marie Congiusta and Analia Veitz-Keenan New York University, College of Dentistry, New York, USA

1. Kersten DD, Mines $P$, Sweet $M$. Use of the microscope in endodontics: results of a questionnaire. J Endod 2008; 34: 804-807.

2. Wong AW, Zhu X, Zhang S, Li SK, Zhang C, Chu CH. Treatment time for nonsurgical endodontic therapy with or without a magnifying loupe. BMC Oral Health 2015; 15: 40. doi:10.1186/s12903-015-0025-7.

3. Del Fabbro M, Taschieri S. Endodontic therapy using magnification devices: a systematic review. J Dent 2010; 38: 269-275.

Evidence-Based Dentistry (2016) 17, 84-85. doi:10.1038/sj.ebd.6401189 